\title{
Utility and safety of three-dimensional contrast low-dose dobutamine echocardiography in the evaluation of myocardial viability early after an acute myocardial infarction
}

Piotr Scisło, Janusz Kochanowski, Łukasz Kołtowski, Grzegorz Opolski

First Chair and Department of Cardiology, Medical University of Warsaw, Warsaw, Poland

Submitted: 16 December 2015

Accepted: 8 January 2016

Arch Med Sci 2018; 14, 3: 488-492

DOI: https://doi.org/10.5114/aoms.2016.58650

Copyright (c 2016 Termedia \& Banach

\section{Abstract}

Introduction: The aim of the study was to determine the utility and safety of three-dimensional contrast low-dose dobutamine echocardiography (3DCLDDE) in the evaluation of myocardial viability early after ST-elevation myocardial infarction (STEMI).

Material and methods: We prospectively evaluated a group of 100 consecutive patients. Myocardial viability was assessed using dobutamine echocardiography in 76 patients with segmental wall motion abnormalities, including 37 patients evaluated using 3DCLDDE and 39 patients evaluated using a standard low-dose dobutamine echocardiography protocol (LDDE), alternately.

Results: Single ventricular ectopic beats were observed during the test in $1(2.5 \%, 1, p=1)$ patient in the 3DCLDDE group, while pain $(1, p=1)$ dyspnea $(1, p=1)$, single ventricular beats $(2, p=1)$, and complex ventricular arrhythmia $(2, p=0.49)$ were noted in $4(10 \%)$ patients in the LDDE group. Five-year survival was $89 \%$ in the 3DCLDDE group and $87 \%$ in the LDDE group.

Conclusions: 3DCLDDE and LDDE are equally safe and useful in patients after STEMI.

Key words: three-dimensional contrast dobutamine stress echocardiography.

\section{Introduction}

Immediate myocardial revascularization is the mainstay of the current management of ST-elevation myocardial infarction (STEMI) patients [1]. This results in a reduction of the extent of infarction or even allows significant myocardial damage to be avoided. Despite modern management, segmental left ventricular wall motion abnormalities are seen in some patients. Although it is usually not necessary to determine prospects of full functional recovery immediately, evaluation of myocardial viability is of key importance in some clinical situations.

A diagnostic test used commonly to evaluate whether post-infarction left ventricular dysfunction is permanent or associated with reversible myocardial stunning or hibernation is low-dose dobutamine echocardi-

\author{
Corresponding author: \\ Piotr Scisło MD, PhD \\ First Chair and \\ Department of Cardiology \\ Medical University of Warsaw \\ 1a Banacha St \\ 02-097 Warsaw, Poland \\ Phone: +48 225992612 \\ Fax: +48 225991234 \\ E-mail: scislo@wum.edu.pl
}


ography (LDDE) [2, 3]. In comparison to other modalities, which may serve the same purpose, including cardiovascular magnetic resonance (CMR) and single-photon emission computed tomography (SPECT) myocardial perfusion scintigraphy, LDDE is less expensive and more available. Compared to other imaging methods and nuclear medicine techniques, technical aspects of LDDE may limit the ability to obtain good quality images (in about $10 \%$ of patients). New techniques based on three-dimensional imaging $[4,5]$ and the use of ultrasound contrast agents improve the diagnostic value of stress echocardiography compared to CMR and SPECT $[6,7]$.

However, the safety of exposing damaged myocardium to large ultrasound beam volumes during contrast-enhanced three-dimensional echocardiography has not been established.

The aim of our study was to compare the utility and safety of three-dimensional contrast lowdose dobutamine echocardiography (3DCLDDE) and the conventional diagnostic LDDE protocol.

\section{Material and methods}

The study was performed in the First Chair and Department of Cardiology, Medical University of Warsaw. The study protocol was approved by the Bioethics Committee at the Medical University of Warsaw. We studied 100 consecutive patients after STEMI, treated according to the Polish and European Society of Cardiology guidelines [1]. The study group included 61 patients after a first myocardial infarction treated invasively. Despite percutaneous coronary intervention, segmental left ventricular wall motion abnormalities were seen in 37 patients at 7 days after revascularization. To determine prospects of left ventricular function recovery in these patients, we evaluated myocardial viability by dobutamine echocardiography between the $7^{\text {th }}$ and $10^{\text {th }}$ day of the hospital stay using the evaluated protocol (3DCLDDE group) that included contrast-enhanced three-dimensional (3D) and two-dimensional (2D) echocardiography. The control group (LDDE) included 39 patients after a myocardial infarction in whom myocardial viability was evaluated by conventional dobutamine stress 2D echocardiography. Alternating allocation was used to assign patients to the 3DCLDDE or LDDE group.

Echocardiographic examinations were performed using a Philips iE33 system with s5-1, x3-1, and $\times 5-1$ probes (Philips, Andover, Massachusetts, USA) and recorded digitally.

According to the study protocol, all patients underwent a complete, typical resting echocardiography. Left ventricular ejection fraction was calculated using the biplane modified Simpson method.
The protocol of dobutamine echocardiography in the 3DCLDDE group included recording of 2D images in the LAX, SAX, AP4C, and AP2C, and 3D images at baseline, at every 5 min of dobutamine infusion at the rate of 5,10 , and $20 \mu \mathrm{g} / \mathrm{kg}$ body weight/min, and at $10 \mathrm{~min}$ after termination of the dobutamine infusion. At baseline and at peak dose, a slow bolus of an echocardiographic contrast agent (sulfur hexafluoride - SonoVue, Bracco) was administered. 2D images were recorded before and after contrast microbubble destruction using a low mechanical index (MI 0.1) ultrasound beam. A high mechanical index ultrasound impulse (Flash, MI 1.2) lasting for 3 cardiac cycles was used to destroy contrast microbubbles and image myocardial perfusion. 3D images were recorded in the full volume mode, and contrast microbubble destruction was obtained by increasing the mechanical index manually to the maximum values.

In the control group the echocardiographic examinations did not include 3D image recording and contrast agent administration, which is a standard for LDDE tests.

Following dobutamine echocardiography, left ventricular wall motion was evaluated using a 17-segment model. The wall motion score index (WMSI) was calculated using the obtained data.

Five-year survival was verified using the electronic PESEL/eWUŚ database of the National Health Fund.

\section{Statistical analysis}

Statistical analysis was performed using the SAS 9.2/JMP 12 software. Quantitative variables were expressed as mean values \pm standard deviation, and qualitative variables as percentages. Normal distribution of the quantitative variables was confirmed using the Kolmogorov-Smirnov test. Between-group comparisons were performed using the Student $t$ test for normally distributed quantitative variables, the Mann-Whitney test for non-normally distributed quantitative variables, and the Fisher test for categorical variables.

\section{Results}

The study and control groups were of a similar size. A non-significantly larger proportion of men was noted in the study group, while patients in the control group were older. The baseline clinical characteristics of both groups were comparable. Five-year survival was $89 \%$ in the 3DCLDDE group and $87 \%$ in the control group (Table I).

Basic cardiac dimensions did not differ between the two groups, except for left atrial size and intraventricular septum diastolic dimension (IVSd), which was slightly but significantly higher in the control group (Table II). 
Table I. Study and control group characteristics

\begin{tabular}{|lccc|}
\hline Parameter & 3DCLDDE & LDDE & $P$-value \\
\hline Age & $\begin{array}{c}56 \pm 11 \\
(95 \% \text { Cl: } 52-60)\end{array}$ & $\begin{array}{c}62 \pm 9.3 \\
(95 \% \text { Cl: } 59-65)\end{array}$ & 0.001 \\
\hline Men & $28(75 \%)$ & $24(61 \%)$ & 0.18 \\
\hline Hypertension & $20(54 \%)$ & $23(59 \%)$ & 0.66 \\
\hline Diabetes & $8(21 \%)$ & $9(22 \%)$ & 0.87 \\
\hline Dyslipidemia & $18(53 \%)$ & $20(51 \%)$ & 0.81 \\
\hline B-blockers & $12(32 \%)$ & $14(35 \%)$ & 0.75 \\
\hline ACEi & $15(40 \%)$ & $17(43 \%)$ & 0.78 \\
\hline STEMI anterior & $25(67 \%)$ & $25(64 \%)$ & 0.75 \\
\hline 5-year survival & $33(89 \%)$ & $34(87 \%)$ & 0.13 \\
\hline
\end{tabular}

Table II. Comparison of baseline echocardiographic parameters

\begin{tabular}{|c|c|c|c|}
\hline Parameter & $\begin{array}{c}\text { 3DCLDDE } \\
\text { Mean, standard deviation } \\
(95 \% \mathrm{Cl})\end{array}$ & $\begin{array}{c}\text { LDDE } \\
\text { Mean, standard deviation } \\
(95 \% \mathrm{Cl})\end{array}$ & $P$-value \\
\hline LVDd [cm] & $5.1 \pm 0.5(4.9-5.2)$ & $5.3 \pm 0.6(5.2-5.6)$ & 0.11 \\
\hline IVSd $[\mathrm{cm}]$ & $1.2 \pm 0.2(1.0-1.2)$ & $1.1 \pm 0.1(1.0-1.1)$ & $<0.05$ \\
\hline PWDt [cm] & $1.0 \pm 0.2(1.0-1.2)$ & $1.0 \pm 0.1(0.9-1.0)$ & 0.58 \\
\hline $\mathrm{RV}[\mathrm{cm}]$ & $2.5 \pm 0.3(2.4-2.6)$ & $2.6 \pm 0.4(2.4-2.7)$ & 0.22 \\
\hline Ao $[\mathrm{cm}]$ & $3.0 \pm 0.5(2.9-3.2)$ & $3.2 \pm 0.5(3.0-3.4)$ & 0.08 \\
\hline $\mathrm{LA}[\mathrm{cm}]$ & $3.6 \pm 0.5(3.4-3.7)$ & $4.1 \pm 0.5(3.9-4.3)$ & 0.03 \\
\hline $\mathrm{EF}(\%)$ & $47 \pm 8.9(43-49)$ & $44 \pm 9.5(41-47)$ & 0.14 \\
\hline
\end{tabular}

Table III. Comparison of dobutamine echocardiography parameters in the two groups

\begin{tabular}{|lccc|}
\hline Parameter & $\begin{array}{c}\text { 3DCLDDE } \\
\text { Mean, standard deviation } \\
\mathbf{( 9 5 \% ~ C l )}\end{array}$ & $\begin{array}{c}\text { Lean, standard deviation } \\
(\mathbf{9 5 \%} \mathrm{Cl})\end{array}$ & P-value \\
\hline WMSI at rest & $1.36 \pm 0.14(1.31-1.41)$ & $1.41 \pm 0.23(1.33-1.48)$ & 0.34 \\
\hline WMSI at peak dose & $1.21 \pm 0.18(1.27-1.15)$ & $1.27 \pm 0.2(1.2-1.34)$ & 0.32 \\
\hline WMSI increase & $0.15 \pm 0.09(0.12-0.18)$ & $0.17 \pm 0.24(0.09-0.25)$ & 0.58 \\
\hline Maximum dobutamine dose & $19.7 \pm 4.6(18-21)$ & $22.7 \pm 7.0(20-25)$ & 0.052 \\
\hline Duration of dobutamine test & $11.3 \pm 2.5(10-12)$ & $12.1 \pm 3.3(11-13)$ & 0.35 \\
\hline Resting heart rate & $68 \pm 10(65-72)$ & $64 \pm 10(60-61)$ & 0.06 \\
\hline Resting blood pressure & $116 \pm 15(111-121) /$ & $126 \pm 12(121-130) /$ & $0.009 / 0.11$ \\
& $67 \pm 9(64-70)$ & $71 \pm 10(68-74)$ & 0.31 \\
\hline Heart rate at peak dose & $82 \pm 18(75-87)$ & $87 \pm 23(79-94)$ & $0.005 / 0.73$ \\
\hline Blood pressure at peak dose & $131 \pm 23(123-138) /$ & $149 \pm 24(141-157) /$ & - \\
\hline $\begin{array}{l}\text { Contrast agent dose per } \\
\text { patient [ml] }\end{array}$ & $70 \pm 12(66-70)$ & $71 \pm 12(67-75)$ & - \\
\hline
\end{tabular}

Both groups were comparable in terms of dobutamine echocardiography parameters (Table III).

Wall motion score index both at rest and at peak dose, WMSI increase, duration of the dobu- tamine test, dobutamine dose, and heart rate and diastolic blood pressure at baseline and at peak workload did not differ significantly between the two groups. Higher systolic blood pressure at rest 
Table IV. Comparison of dobutamine echocardiography adverse effects in the two groups

\begin{tabular}{|lccc|}
\hline Parameter & 3DCLDDE & LDDE & $P$-value \\
\hline SVPB & 0 & 0 & NA \\
\hline Single VPB & 1 & 2 & 1 \\
\hline Complex VPB & 0 & 1 & 0.49 \\
\hline Dyspnea & 0 & 1 & 1 \\
\hline Chest pain & 0 & & 1 \\
\hline
\end{tabular}

and at peak dobutamine dose was seen in the control group.

Single ventricular ectopic beats were observed during dobutamine testing in 1 patient in the study group. In the control group, chest pain with a rise in blood pressure was seen in 1 patient, complex ventricular arrhythmia was noted in 2 patients, and single ectopic beats were observed in another 2 patients (Table IV).

\section{Discussion}

Low-dose dobutamine echocardiography has been used for many years to identify viable myocardium in patients with post-infarction left ventricular dysfunction [8]. Many authors indicate that this technique is relatively safe and associated with a low risk of complications [9]. Similar conclusions may be arrived at based on our findings.

However, LDDE has some ultrasonographic limitations due to not always ideal tissue ultrasound translucency and the quality of acoustic windows used to obtain typical echocardiographic views. Echocardiographic contrast agents that were introduced more than 20 years ago allowed endocardial visualization and evaluation of tissue perfusion to be improved [10-12] even in patients with technically difficult imaging conditions.

After several years of use of these agents, questions arose regarding their safety. A few reports were published describing significant complications that might have (but also might not have) been related to administration of echocardiographic contrast agents. Based on these reports, warnings on the use of echocardiographic contrast agents were released by drug regulatory agencies in 2007 (FDA - www.fda.gov; EMA www.ema.europa.eu).

However, as early as in 2008 Wei et al. [13] performed a retrospective multicenter study to evaluate infusion of about 80,000 doses of echocardiographic contrast agents (Optison, Definity). This analysis included 10,000 cases in which these agents were administered to critically ill patients, and about $28 \%$ of contrast-enhanced studies were stress tests. The only adverse effects definitely associated with administration of a contrast agent were anaphylactic reactions in 4 (0.006\%) patients, and 8 patients presented symptoms that might have been associated with administration of a contrast agent. Senior et al. arrived at similar conclusions regarding safety of the SonoVue contrast agent [14]. Following publication of many similar reports, absolute contraindications to the use of contrast agents in patients after a myocardial infarction were withheld, and cardiac societies allowed their use [15].

In our study, similarly to large multicenter analyses, we noted a very low rate of adverse events that might have been, but was not necessarily, directly associated with the use of a contrast agent.

In the study group, one element of the study protocol was to perform a three-dimensional recording of contrast agent flow. A short ultrasound impulse (flash) was used to reset the perfusion image by contrast microbubble destruction, resulting in their content being released to the coronary circulation. This situation occurs typically during two-dimensional contrast myocardial perfusion echocardiography, which is considered a safe diagnostic technique [16-19], even when taking into account potential genotoxic changes [20]. In our study, however, we used a three-dimensional ultrasound beam which increased the amount of the contrast agent undergoing microbubble destruction at the same time. This potentially increases the amount of contrast agent reaching dysfunctional myocardium. However, our study showed that three-dimensional contrast echocardiography is safe even in patients after a recent acute coronary event.

Although the patients in the LDDE group were slightly older, this should not have an impact on the study results [21]. The septum in diastole was slightly thicker in the study group, whereas the left atrial dimension was slightly larger in the control group. These observations did not correlate with the prevalence of hypertension, which was comparable in both groups. The baseline systolic blood pressure was in the normal range in both groups, but it was higher in the control group. Peak dose dobutamine systolic pressure was slightly higher in the control group. However, these observations were in the expected range [22]. 
Due to the high cost of dedicated contrast study equipment, a dedicated infusion pump was not used for administration of a contrast agent.

The study was performed in a single cardiology unit, which resulted in a reduced capability to recruit patients due to an increased availability of percutaneous coronary revascularization.

Five-year survival in patients evaluated using the study protocol was $89 \%$, slightly higher compared to the control group (87\%), which may indirectly indicate that clinical management decisions based on these tests were appropriate.

In conclusion, use of small amounts of a contrast agent that are sufficient to opacify the left ventricle and evaluate myocardial perfusion does not increase the risk of adverse effects during three-dimensional contrast-enhanced stress dobutamine echocardiography in hemodynamically stable patients early after an acute myocardial infarction.

\section{Acknowledgments}

The study was supported by a grant from the Ministry of Scientific Research and Information Technology (NN402 04831/1523).

\section{Conflict of interest}

The authors declare no conflict of interest.

\section{References}

1. Steg PG, James SK, Atar D, et al.; ESC guidelines for the management of acute myocardial infarction in patients presenting with ST-segment elevation. The Task Force on the management of ST-segment elevation acute myocardial infarction of the European Society of Cardiology (ESC). Eur Heart J 2012; 33: 2569-619.

2. Bax JJ, Poldermans D, Visser FC, et al. Delayed recovery of hibernating myocardium after surgical revascularization: implications for discrepancy between metabolic imaging and dobutamine echocardiography for assessment of myocardial viability. J Nucl Cardiol 1999; 6: 685-7.

3. Ay T, Pasquet A, Robert A, et al. Usefulness of low-dose dobutamine echocardiography for selection of patients with severely depressed left ventricular function for coronary bypass grafting. Am J Cardiol 2002; 90: 323-5.

4. Lang RM, Badano LP, Tsang W, et al. EAE/ASE recommendations for image acquisition and display using three-dimensional echocardiography. Eur Heart J Cardiovasc Imaging 2012; 13: 1-46.

5. Pulerwitz T, Hirata K, Abe $\mathrm{Y}$, et al. Feasibility of using a real-time 3-dimensional technique for contrast dobutamine stress echocardiography. J Am Soc Echocardiogr 2006; 19: 540-5.

6. Lipiec P, Wejner-Mik P, Krzemińska-Pakuła M, et al. Accelerated stress real-time myocardial contrast echocardiography for the detection of coronary artery disease: comparison with $99 \mathrm{mtc}$ single photon emission computed tomography. J Am Soc Echocardiogr 2008; 21: 941-7.

7. Salerno M, Beller GA. Noninvasive assessment of myocardial perfusion. Circ Cardiovasc Imaging 2009; 2: 412-24.

8. Salustri A, Elhendy A, Garyfallydis P, et al. Prediction of improvement of ventricular function after first acute myocardial infarction using low-dose dobutamine stress echocardiography. Am J Cardiol 1994; 74: 853-6.

9. Picano E, Mathias W Jr, Pingitore A, Bigi R, Previtali M. Safety and tolerability of dobutamine-atropine stress echocardiography: a prospective, multicentre study. Lancet 1994; 344: 1190-2.

10. Wejner-Mik P, Lipiec P, Kasprzak JD. Long-term prognostic value of dipyridamole stress myocardial contrast echocardiography. Eur J Echocardiogr 2011; 12: 762-6.

11. Huang WC, Chiou KR, Liu CP, et al. Comparison of real-time contrast echocardiography and low-dose dobutamine stress echocardiography in predicting the left ventricular functional recovery in patients after acute myocardial infarction under different therapeutic intervention. Int J Cardiol 2005; 104: 81-91.

12. Hillis GS, Mulvagh SL, Pellikka PA, et al. Comparison of intravenous myocardial contrast echocardiography and low-dose dobutamine echocardiography for predicting left ventricular functional recovery following acute myocardial infarction. Am J Cardiol 2003; 92: 504-8.

13. Wei K, Mulvagh SL, Carson L, et al. The safety of definity and optison for ultrasound image enhancement: a retrospective analysis of 78,383 administered contrast doses. J Am Soc Echocardiogr 2008; 21: 1202-6.

14. Senior R, Moreo A, Gaibazzi N, et al. Comparison of sulfur hexafluoride microbubble (sonovue)-enhanced myocardial contrast echocardiography with gated single-photon emission computed tomography for detection of significant coronary artery disease: a large European Multicenter Study. J Am Coll Cardiol 2013; 62: 1353-61.

15. Porter TR, Abdelmoneim S, Belcik JT, et al. Guidelines for the cardiac sonographer in the performance of contrast echocardiography: a focused update from the American Society of Echocardiography. J Am Soc Echocardiogr 2014; 27: 797-810.

16. Tsutsui JM, Elhendy A, Xie F, O'Leary EL, McGrain AC, Porter TR. Safety of dobutamine stress real-time myocardial contrast echocardiography. J Am Coll Cardiol 2005; 45: 1235-42.

17. Timperley J, Mitchell ARJ, Thibault H, Mirza IH, Becher H. Safety of contrast dobutamine stress echocardiography: a single center experience. J Am Soc Echocardiogr 2005; 18: 163-7.

18. Timperley J, Becher H. Safety of dobutamine contrast stress echocardiography. J Am Coll Cardiol 2005; 46: 1963.

19. Gaibazzi N, Silva L, Reverberi C. Safety and positive predictive value of high-dose dipyridamole stress-echocardiography with or without contrast flash-replenishment perfusion imaging in patients with suspected or known coronary artery disease. Int J Cardiol 2012; 154: 382-3.

20. Gaibazzi N, Marziliano N, Porter TR, et al. Assessment of DNA damage associated with standard or contrast diagnostic echocardiography. Int J Cardiol 2015; 180: 96-9.

21. Bernheim AM, Kittipovanonth M, Takahashi PY, Gharacholou SM, Scott CG, Pellikka PA. Does the prognostic value of dobutamine stress echocardiography differ among different age groups? Am Heart J 2011; 161: 740-5.

22. Abram S, Arruda-Olson AM, Scott CG, et al. Typical blood pressure response during dobutamine stress echocardiography of patients without known cardiovascular disease who have normal stress echocardiograms. Eur Heart J Cardiovasc Imaging 2015 Jul 22. pii: jev165. [Epub ahead of print]. 